\title{
Autofocus Bayesian Compressive Sensing for Multipath Exploitation in Urban Sensing
}

\author{
Qisong Wu, Yimin D. Zhang, Moeness G. Amin, and Fauzia Ahmad \\ Center for Advanced Communications, Villanova University, Villanova, PA 19085, USA
}

\begin{abstract}
Exploitation of group sparsity under multipath propagation enables high-resolution ghost-free imaging in urban sensing and throughthe-wall radar imaging applications. Multipath exploitation schemes typically require exact prior information of the indoor scene layout and transceiver locations to eliminate ghosts targets. Imperfections in the prior knowledge lead to performance degradation of such schemes. In this paper, a novel autofocus Bayesian compressive sensing approach is proposed for joint scene reconstruction and correction of phase errors resulted from transceiver position uncertainties. Supporting simulation results are provided to demonstrate the effectiveness of the proposed algorithm.

Index Terms-Through-the-wall radar imaging, autofocus, multipath
\end{abstract} exploitation, Bayesian compressive sensing.

\section{INTRODUCTION}

A major challenge in urban sensing and through-the-wall radar imaging (TWRI) is the presence of multipath. Multipath returns originate from reflections of electromagnetic (EM) waves off the targets of interest in conjunction with the building walls, floor, and/or ceiling. Such secondary reflections gives rise to false targets or "ghosts" at positions other than those of the actual targets, which clutter the imaged scene and compromise target detection [1-5]. If precise knowledge of the room layout and transceiver locations is available a priori, the multipath returns can be exploited to improve the imaging performance [6-9]. However, as the transceivers are often hand-held or mounted on moving platforms, it is inevitable to introduce transceiver location errors in practical operational scenarios. Therefore, it is imperative to develop autofocus multipath exploitation techniques that can provide accurate imaging in the presence of location uncertainties.

Various studies have been presented for autofocus problems in radar imaging applications. One of the most well-known techniques, i.e., phase gradient autofocus (PGA) [10], estimates the phase error using the data obtained by isolating many single defocused targets via center-shifting and windowing operations. It is based on the assumption that there exist prominent scatterers in the range cell. An alternate technique is based on the optimization of a sharpness metric of the defocussed image intensity $[11,12]$. However, these conventional techniques are invalid in TWRI, because it is difficult to distinguish the ghost targets from the true targets, especially when the targets of interest are stationary. Recently developed compressive sensing (CS) based autofocus approaches $[13,14]$ form a different class of effective tools for sparse signal reconstruction in the presence of phase errors. Both approaches, which introduce a diagonal matrix to model the phase errors, involve an iterative scheme including consecutive steps of sparse signal recovery and phase error estimation.

In this paper, inspired by [13, 14], a novel autofocus Bayesian compressive sensing (ABCS) scheme is proposed to achieve highresolution imaging with multipath exploitation in the presence of uncertain transceiver locations in TWRI. To the best of our knowledge, autofucus imaging with multipath exploitation has not been investigated previously. We first develop a forward model under multipath propagation in the presence of imperfect transceiver locations.
It is observed that the phase errors generally differ across multipath propagations, whereas all the pixels share similar phase errors in each path, due to the relative large propagation distance and finite size of the region of interest. Based on this forward model, a novel autofocus imaging approach is proposed to jointly acquire high-resolution image reconstruction and implement the phase error corrections within the nonparametric sparse Bayesian learning framework. Simulation results verify the effectiveness of the forward model and superiority of the proposed ABCS method.

Notations: We use lower-case (upper-case) bold characters to denote vectors (matrices). $p(\cdot)$ denotes the probability density function (pdf). $\mathcal{C N}(x \mid a, b)$ represents a random variable $x$ that follows a complex Gaussian distribution with mean $a$ and variance $b$, and $\mathrm{U}(x \mid a, b)$ describes that random variable $x$ is uniform distributed between $a$ and $b .\lceil x / y\rceil$ denotes the modulus after $x$ over $y$, and $\lfloor x / y\rfloor$ denotes the remainder after $x$ over $y$. In addition, $(\cdot)^{T}$ and $(\cdot)^{H}$, respectively, denote transpose and conjugate transpose of a matrix or vector, $\mathbf{I}_{N}$ denotes the $N \times N$ identity matrix. " $\odot$ " and " $\otimes$ " denote element-wise (Hadamard) and kronecker multiplication, respectively. $\operatorname{Im}(x)$ and $\operatorname{Re}(x)$ are respectively the imaginary and real components of $x$.

\section{SIGNAL MODEL}

We assume monostatic stepped-frequency radar operation [3] and consider a few stationary targets enclosed by two side walls and a back wall. For simplicity, the front wall is ignored. Extensive studies are available on the parameter estimation and compensation of the front wall $[1,15,16]$. A wideband transceiver is mounted on a vehicle moving parallel to the $x$-axis at downrange $y_{0}$ to synthesize an $N$-element aperture, and transmits a stepped-frequency signal consisting of $M$ frequencies at each position. The $M$ frequencies, $f_{m}$, $m \in\{1, \cdots, M\}$, are uniformly spaced over the signal bandwidth $B$. Under monostatic operation, the target returns can be expressed in baseband as [8],

$$
\begin{aligned}
y(m, n) & =\sum_{l=1}^{N_{x} N_{y}} w_{l} \exp \left(-j 2 \pi \frac{r_{l n}}{\lambda_{m}}\right), \\
r_{l n} & =2 \sqrt{\left(x_{l}-x_{n}\right)^{2}+\left(y_{l}-y_{0}\right)^{2}},
\end{aligned}
$$

where $N_{x}$ and $N_{y}$, respectively, denote the number of pixels in crossrange and downrange within the region of interest, $r_{l n}$ represents the round-trip propagation distance between the $l$ th scatterer and $n$th transceiver position, $w_{l}$ is the complex reflectivity corresponding to the $l$ th pixel, and $\lambda_{m}=f_{m} / c$ with $c$ being the speed of light. The location of the $n$th synthetic aperture position is denoted by $\left(x_{n}, y_{0}\right)$.

The measurement data vector $\mathbf{y}=[y(1,1), \cdots, y(M, N)]^{T} \in$ $\mathcal{C}^{M N}$ is obtained by stacking all measurements $y(m, n)$ into a single column vector, i.e.,

$$
\mathbf{y}=\mathbf{D} \mathbf{w},
$$




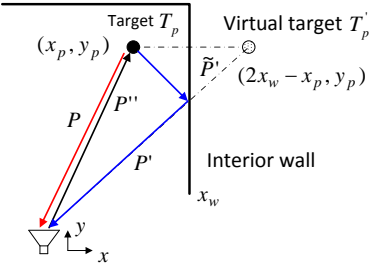

(a)

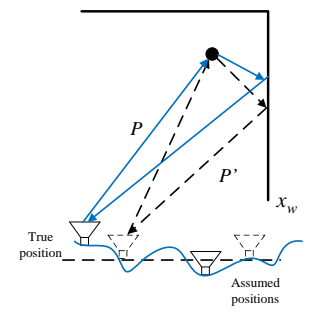

(b)
Fig. 1: (a) Multipath propagations via reflections at interior walls. (b) Multipath propagation with transceiver position errors.

with the $(i, l)$ th element of the sensing matrix $\mathbf{D} \in \mathcal{C}^{M N \times N_{x} N_{y}}$ given by

$$
[\mathbf{D}]_{i l}=\exp \left(-j 2 \pi \frac{r_{l n}}{\lambda_{m}}\right)
$$

for $i \in\{1, \cdots, M N\}, m=\lceil i / M\rceil$, and $n=\lfloor i / N\rfloor$, and $\mathbf{w} \in$ $\mathcal{C}^{N_{x} N_{y}}$ represents the target reflectivity vector. Note that most of elements in $\mathbf{w}$ are zero due to limited spatial occupancies in practice. As such, CS can be used for sparse signal reconstruction.

\section{A. Interior Wall Multipath}

The transmitted signal may reach the target via reflections at one or more secondary reflectors (typically an interior wall), as depicted in Fig. 1(a). Assuming one direct path and a maximum of $K-1$ multipath returns, the forward model under multipath propagation can be expressed as [8],

$$
\mathbf{y}=\mathbf{D}_{0} \mathbf{w}_{0}+\mathbf{D}_{1} \mathbf{w}_{1}+\cdots+\mathbf{D}_{K-1} \mathbf{w}_{K-1},
$$

where $\mathbf{D}_{0} \in \mathcal{C}^{M N \times N_{x} N_{y}}$ and $\mathbf{w}_{0} \in \mathcal{C}^{N_{x} N_{y}}$, respectively, denote the sensing matrix and complex reflectivities for the direct path (defined in eqs. (3) and (4)), while $\mathbf{D}_{k}$ and $\mathbf{w}_{k}$ with $k \in\{1, \cdots K-1\}$ are the respective sensing matrix and the complex reflectivities corresponding to the $k$ th multipath. The elements of $\mathbf{D}_{k}, k \in\{1, \cdots K-1\}$, are phase terms, analogous to (4), which incorporate the propagation distances associated with the $k$ th multipath return. Assuming knowledge of the room geometry, these distances can be readily computed from geometric considerations $[7,8]$. The target reflectivity vectors $\mathbf{w}_{k}, k=0, \ldots, K-1$, share a common support, i.e., they have the same respective positions of nonzero entries, although the exact values of the reflectivity coefficients generally differ [8].

\section{B. Multipath Propagation with Uncertain Transceiver Positions}

Assuming $\left(\Delta x_{n}, \Delta y_{n}\right)$ to be the uncertainties in the $n$th transceiver position, the propagation distance between the $n$th transceiver and the $l$ th scatterer for the direct path is expressed as,

$$
\begin{aligned}
\tilde{r}_{l n} & =2 \sqrt{\left(x_{l}-x_{n}-\Delta x_{n}\right)^{2}+\left(y_{l}-y_{0}-\Delta y_{n}\right)^{2}} \\
& \approx r_{l n}-2 \sin \theta_{l n} \Delta x_{n}-2 \cos \theta_{l n} \Delta y_{n} \\
& =r_{l n}-2 \Delta r_{n} \sin \left(\theta_{n l}+\Delta \theta_{n}\right), \\
\Delta r_{n} & =\sqrt{\Delta x_{n}^{2}+\Delta y_{n}^{2}}, \quad \Delta \theta_{n}=\arccos \left(\Delta x_{n} / \Delta r_{n}\right),
\end{aligned}
$$

where $2 \Delta r_{n} \sin \left(\theta_{n l}+\Delta \theta_{n}\right)$ denotes the deviation from the hypothesized propagation distance $r_{l n}$ under $\Delta x_{n} \ll r_{l n}$ and $\Delta y_{n} \ll r_{l n}$. Considering the finite room size and relatively large $r_{l n}$, Eq. (6) can be further approximated as,

$$
\tilde{r}_{l n} \approx r_{l n}-2 \Delta r_{n} \sin \left(\theta_{n}+\Delta \theta_{n}\right),
$$

where $\theta_{n}$ is the squint angle from the $n$th hypothesized transceiver position to a reference scatterer in the observed scene. The phase errors generally differ for each multipath due to the diverse propagation geometries. As such, the forward model under multipath propagation in the presence of uncertain transceiver positions is given by,

$$
\mathbf{y}=\mathbf{A}_{0} \mathbf{D}_{0} \mathbf{w}_{0}+\mathbf{A}_{1} \mathbf{D}_{1} \mathbf{w}_{1}+\cdots+\mathbf{A}_{K-1} \mathbf{D}_{K-1} \mathbf{w}_{K-1},
$$

where $\mathbf{A}_{k}$ denotes the phase error matrix for the $k$ th multipath, which is determined by the frequency and the path errors and is given as

$$
\mathbf{A}_{k}=\operatorname{diag}\left(\mathbf{e}_{k}\right), \quad \mathbf{e}_{k}=\left[e^{j \psi_{1}^{(k)}}, \cdots, e^{j \psi_{M N}^{(k)}}\right]^{T}
$$

\section{Compressed Data Collection}

Within the CS framework, we perform sparse reconstruction using only a subset of the full measurements. Mathematically, the data reduction operation can be expressed as a measurement or downsampling matrix $\mathbf{B} \in \mathcal{R}^{L \times M N}$ acting on the full measurements, where $L \ll M N$ is the number of reduced measurements. For stepped frequency radar system, a binary measurement matrix $\mathbf{B} \in\{0,1\}^{L \times M N}$ is a reasonable choice $[8,17]$. As such, we acquire an undersampled measurement vector $\mathbf{s} \in \mathcal{C}^{L}$ as

$$
\mathbf{s}=\mathbf{B y}=\mathbf{E}_{0} \mathbf{D}_{0} \mathbf{w}_{0}+\mathbf{E}_{1} \mathbf{D}_{1} \mathbf{w}_{1}+\cdots+\mathbf{E}_{K-1} \mathbf{D}_{K-1} \mathbf{w}_{K-1}+\boldsymbol{\epsilon},
$$

where $\mathbf{E}_{k}=\mathbf{B} \mathbf{A}_{k} \in \mathcal{C}^{L \times M N}$. Without loss of generality, a measurement noise vector $\epsilon \in \mathcal{C}^{L}$ is added to the reduced data.

\section{Autofocus Bayesian Compressive Sensing}

\section{A. Generative model}

From (11), the following three observations are in order: 1) Direct and multipath returns may be unresolvable; 2) The reflectivity coefficients share the same sparsity pattern across multipath propagations; and 3) There exist separate phase errors for each multipath. These unique properties are utilized to propose a novel ABCS method below.

Following the sparse Bayesian learning technique, we place a hierarchical structure on $\mathbf{W}=\left[\mathbf{w}_{0}, \cdots, \mathbf{w}_{K-1}\right]$ to encourage group sparsity:

$$
\begin{aligned}
p(\mathbf{W} \mid \boldsymbol{\alpha}) & =\prod_{i=1}^{N_{x} N_{y}} \mathcal{C N}\left(\mathbf{w}_{i} \cdot \mid, \mathbf{0}, \alpha_{i} \mathbf{I}_{K}\right) \\
& =\prod_{i=1}^{N_{x} N_{y}} \prod_{k=0}^{K-1} \mathcal{C N}\left(w_{k i} \mid 0, \alpha_{i}\right),
\end{aligned}
$$

where $\mathbf{w}_{i} .=\left[w_{i, 0}, \cdots, w_{i, K-1}\right] \in \mathcal{C}^{1 \times K}$ is the reflectivity coefficient collection corresponding to the $i$ th scatterer across the $K$ propagation paths, and $\alpha_{i}$ is the variance shared by each element of $\mathbf{w}_{i}$.. When $\alpha_{i}$ approaches zero, its corresponding element in $\mathbf{w}_{i}$. will approach zero and be pruned away from the model.

To promote sparsity over the reflectivity coefficients $\mathbf{w}_{i}$, a Gamma prior is placed on $\alpha_{i}^{-1}$ [18], i.e.,

$$
\alpha_{i}^{-1} \sim \operatorname{Gamma}\left(\alpha_{i}^{-1} \mid a, b\right),
$$

where

$$
\operatorname{Gamma}\left(x^{-1} \mid a, b\right)=\Gamma(a)^{-1} b^{a} x^{-(a-1)} e^{-\frac{b}{x}},
$$

and $\Gamma(\cdot)$ denotes the Gamma function.

Without loss of generality, a complex Gaussian prior is placed on the additive measurement noise, i.e.,

$$
\boldsymbol{\epsilon} \sim \mathcal{C N}\left(\boldsymbol{\epsilon} \mid 0, \beta_{0}\right)
$$


where $\beta_{0}$ is the noise variance. Similar to $\alpha$, we place $\beta_{0}^{-1}$ on the Gamma prior with parameters $c$ and $d$. We use $a=b=c=d=0$ as a default choice [18].

A noninformative prior is placed on the stochastic error phase as

$$
\psi_{l}^{(k)} \sim \mathrm{U}\left(\psi_{l}^{(k)} \mid 0,2 \pi\right)
$$

with $k \in[1, \cdots, K]$ and $l \in[1, \cdots, L]$.

According to the Bayesian theorem, the posterior distribution can be expressed as,

$$
p\left(\overline{\mathbf{w}}, \boldsymbol{\alpha}, \beta_{0}, \boldsymbol{\Psi} \mid \mathbf{s}\right)=\frac{p\left(\mathbf{s} \mid \overline{\mathbf{w}}, \boldsymbol{\Psi}, \beta_{0}\right) p(\overline{\mathbf{w}} \mid \boldsymbol{\alpha}) p(\boldsymbol{\alpha}) p\left(\beta_{0}\right) p(\mathbf{\Psi})}{p(\mathbf{s})},
$$

where $\overline{\mathbf{w}}=\left[\mathbf{w}_{1}^{T}, \cdots, \mathbf{w}_{N_{x} N_{y}}^{T}.\right] \in \mathcal{C}^{K N_{x} N_{y}}$ is an aligned reflectivity coefficient vector, and $\boldsymbol{\Psi}=\left[\mathbf{e}_{0}, \cdots, \mathbf{e}_{K-1}\right] \in \mathcal{C}^{L \times K}$. The computation of $p(\mathbf{s})$ is often intractable due to the multidimensional integral. We introduce a Monte Carlo Markov Chain (MCMC) method to perform the posterior inference [19].

\section{B. Bayesian Inference Based on MCMC}

In this section, we perform the posterior inference based on the MCMC sampling scheme, which suggests an iteration procedure. We can acquire analytical posterior distribution of $\mathbf{W}$, given other parameters $\left\{\boldsymbol{\alpha}, \beta_{0}, \boldsymbol{\Psi}\right\}$, due to the conjugacy. The effective bound optimization technique is introduced to perform the point estimation for $\boldsymbol{\alpha}$ and $\beta_{0}$. The phase error can be estimated by the maximum a posterior (MAP) technique.

1) Updating $\overline{\mathbf{w}}$ : The posterior distribution of $\overline{\mathbf{w}}$ can be analytically obtained due to the conjugate property. Given $\boldsymbol{\alpha}, \beta_{0}$ and $\boldsymbol{\Psi}$, we have

$$
\begin{aligned}
\mathbf{w}_{i} \cdot & \sim \mathcal{C N}\left(\mathbf{w}_{i \cdot} \mid \boldsymbol{\mu}_{i .}, \boldsymbol{\Sigma}_{i}\right), \quad 1 \leq i \leq N_{x} N_{y}, \\
\boldsymbol{\mu}_{i \cdot} & =\beta_{0}^{-1} \boldsymbol{\Sigma}_{i} \tilde{\mathbf{D}}_{i}^{H} \mathbf{s}, \\
\boldsymbol{\Sigma}_{i} & =\left(\beta_{0}^{-1} \tilde{\mathbf{D}}_{i}^{H} \tilde{\mathbf{D}}_{i}+\alpha_{i}^{-1} \mathbf{I}_{K}\right)^{-1}, \\
\tilde{\mathbf{D}}_{i} & =\left[\mathbf{E}_{0} \mathbf{d}_{0, i}, \cdots, \mathbf{E}_{K-1} \mathbf{d}_{K-1, i}\right],
\end{aligned}
$$

where $\tilde{\mathbf{D}}_{i} \in \mathcal{C}^{L \times K}$ denotes the collection of the $i$ th column across the $K$ matrices $\left\{\mathbf{E}_{k} \mathbf{D}_{k}\right\}_{k=0}^{K-1}$, and $\mathbf{d}_{k, i}$ is the $i$ th column in $\mathbf{D}_{k}$.

2) Updating $\boldsymbol{\alpha}$ and $\beta_{0}$ : With known $\boldsymbol{\alpha}$ and $\beta_{0}$, the mean and covariance of each scattering coefficient are respectively derived in (19) and (20). The associated learning problem, thus, becomes a search for the parameters $\alpha$ and $\beta_{0}$. The empirical Bayesian estimate for $\boldsymbol{\alpha}$ and $\beta_{0}$ is determined by maximizing the marginal likelihood, or equivalently, its logarithm,

$$
\left\{\boldsymbol{\alpha}, \beta_{0}\right\}=\underset{\boldsymbol{\alpha}, \beta_{0}}{\operatorname{argmax}} \mathcal{L}\left(\boldsymbol{\alpha}, \beta_{0}\right)
$$

where

$$
\begin{aligned}
\mathcal{L}\left(\boldsymbol{\alpha}, \beta_{0}\right) & =\sum_{l=1}^{L} \log p\left(\mathbf{s} \mid \boldsymbol{\alpha}, \beta_{0}\right)=\int p\left(\mathbf{s} \mid \overline{\mathbf{w}}, \beta_{0}\right) p(\overline{\mathbf{w}} \mid \boldsymbol{\alpha}) d \overline{\mathbf{w}} \\
& \equiv-\left[\log |\mathbf{C}|+\mathbf{s}^{H} \mathbf{C}^{-1} \mathbf{s}\right] \\
\mathbf{C} & =\beta_{0} \mathbf{I}_{L}+\overline{\mathbf{D}}\left(\mathbf{A} \otimes \mathbf{I}_{K}\right) \overline{\mathbf{D}}^{H} \\
\overline{\mathbf{D}} & =\left[\tilde{\mathbf{D}}_{1}, \cdots, \tilde{\mathbf{D}}_{N_{x} N_{y}}\right]
\end{aligned}
$$

in which $\mathbf{C} \in \mathcal{C}^{L \times L}$, and $\overline{\mathbf{D}} \in \mathcal{C}^{L \times K N_{x} N_{y}}$ with $L \ll K N_{x} N_{y}$. A type-II maximum likelihood (ML) approximation [20] employs the point estimates for $\beta_{0}$ and $\boldsymbol{\alpha}$ to maximize (22), which can be implemented via the expectation maximization (EM) algorithm. However, the method based on EM technique has slow convergence [18]. Several effective approaches have been proposed to speed up the convergence $[18,21,22]$. It is proved that the approach based on the bound optimization technique (known as Majorization-Minimization method) can be used to achieve better sparse solutions [22-24].

In this paper, we derive $\boldsymbol{\alpha}$ by following the bound optimization technique. We use the supporting hyperplane of the second term $\log |\mathbf{C}|$ in (23). Let $\boldsymbol{\alpha}^{*}$ be a given point in the $\boldsymbol{\alpha}$-space. Then,

$$
\begin{aligned}
\mathcal{L}(\boldsymbol{\alpha}) \triangleq & \log |\mathbf{C}|=\log \left|\beta_{0} \mathbf{I}_{L}+\overline{\mathbf{D}}\left(\mathbf{A} \otimes \mathbf{I}_{K}\right) \overline{\mathbf{D}}^{H}\right| \\
& \leq \log \left|\mathbf{C}^{*}\right|+\sum_{i=1}^{N_{x} N_{y}} \operatorname{Tr}\left[\left(\mathbf{C}^{*}\right)^{-1} \tilde{\mathbf{D}}_{i} \tilde{\mathbf{D}}_{i}^{H}\right]\left(\alpha_{i}-\alpha_{i}^{*}\right) \\
& \triangleq \tilde{\mathcal{L}}(\boldsymbol{\alpha})
\end{aligned}
$$

where

$$
\begin{aligned}
& \mathbf{C}^{*}=\beta_{0} \mathbf{I}_{L}+\overline{\mathbf{D}}\left(\mathbf{A}^{*} \otimes \mathbf{I}_{K}\right) \overline{\mathbf{D}}^{H}, \\
& \mathbf{A}^{*}=\operatorname{diag}\left(\alpha_{1}^{*}, \cdots, \alpha_{N_{x} N_{y}}^{*}\right) .
\end{aligned}
$$

Note that the function $\tilde{\mathcal{L}}(\boldsymbol{\alpha})$ defined in (25) is convex over $\boldsymbol{\alpha}$ and, when $\boldsymbol{\alpha}=\boldsymbol{\alpha}^{*}$, we have $\mathcal{L}\left(\boldsymbol{\alpha}^{*}\right)=\tilde{\mathcal{L}}\left(\boldsymbol{\alpha}^{*}\right)$. Further, for any $\boldsymbol{\alpha}_{\text {min }}$ which minimizes $\tilde{\mathcal{L}}(\boldsymbol{\alpha})$, we have $\mathcal{L}\left(\boldsymbol{\alpha}_{\text {min }}\right) \leq \tilde{\mathcal{L}}\left(\boldsymbol{\alpha}_{\text {min }}\right) \leq \tilde{\mathcal{L}}\left(\boldsymbol{\alpha}^{*}\right)$ [23]. Furthermore, based on the identity property, we have a surrogate function for the third term in (23), expressed as

$$
\mathbf{s}^{H} \mathbf{C}^{-1} \mathbf{s} \equiv \min _{\overline{\mathbf{w}}} \beta_{0}^{-1}\|\mathbf{s}-\overline{\mathbf{D}} \overline{\mathbf{w}}\|_{2}^{2}+\overline{\mathbf{w}}^{H}\left(\mathbf{A}^{-1} \otimes \mathbf{I}_{K}\right) \overline{\mathbf{w}}
$$

Substituting (25) and (28) in (23), we can define a new function as

$$
\begin{aligned}
\mathcal{G}(\boldsymbol{\alpha}, \overline{\mathbf{w}}) & \triangleq \beta_{0}^{-1}\left\|\mathbf{s}-\sum_{i=1}^{N_{x} N_{y}} \tilde{\mathbf{D}}_{i} \mathbf{w}_{i}^{T}\right\|_{2}^{2}+\sum_{i=1}^{N_{x} N_{y}} \alpha_{i}^{-1} \mathbf{w}_{i} \cdot \mathbf{w}_{i}^{H} \\
& +\log \left|\mathbf{C}^{*}\right|+\sum_{i=1}^{N_{x} N_{y}} \operatorname{Tr}\left[\left(\mathbf{C}^{*}\right)^{-1} \tilde{\mathbf{D}}_{i} \tilde{\mathbf{D}}_{i}^{H}\right]\left(\alpha_{i}-\alpha_{i}^{*}\right) .
\end{aligned}
$$

It is important to note that $\mathcal{G}(\boldsymbol{\alpha}, \overline{\mathbf{w}})$ is convex in both $\left\{\mathbf{w}_{\tilde{N}}\right\}_{i=1, L}$ and $\boldsymbol{\alpha}$. It is easily shown that the solution $\boldsymbol{\alpha}$ of $\tilde{\mathcal{L}}(\boldsymbol{\alpha})$ in (25) is the solution $\left\{\mathbf{w}_{i}\right\}_{i=1, L}$ of $\mathcal{G}(\boldsymbol{\alpha}, \overline{\mathbf{w}})$ in (29). Thus, $\mathcal{G}(\boldsymbol{\alpha}, \overline{\mathbf{w}})$ is our final cost function.

Setting the derivative of $\mathcal{G}$ with respect to $\alpha_{i}$ to zero, we obtain

$$
\alpha_{i}^{\text {(new) }}=\sqrt{\frac{\mathbf{w}_{i} \cdot \mathbf{w}_{i .}^{H}}{\operatorname{Tr}\left[\left(\mathbf{C}^{*}\right)^{-1} \tilde{\mathbf{D}}_{i} \tilde{\mathbf{D}}_{i}^{H}\right]}} .
$$

It is proved that the updated formulation in (30) based on this bound optimization technique requires far fewer iterations than the one used in the EM algorithm [18, 22, 23]. We acquire the closed-form $\boldsymbol{\alpha}$ by solving a surrogate convex function. Furthermore, we take the derivative of (23) with respect to $\beta_{0}$, and acquire [18],

$$
\begin{aligned}
\beta_{0}^{(\text {new })} & =\frac{\left\|\mathbf{s}-\overline{\mathbf{D}} \overline{\mathbf{w}}^{*}\right\|_{2}^{2}+\operatorname{Tr}\left(\boldsymbol{\Sigma}_{0} \overline{\mathbf{D}} \overline{\mathbf{D}}^{H}\right)}{L}, \\
\overline{\mathbf{w}}^{*} & =\beta_{0}^{-1} \boldsymbol{\Sigma}_{0} \overline{\mathbf{D}}^{H} \mathbf{s}, \\
\boldsymbol{\Sigma}_{0} & =\left(\beta_{0}^{-1} \overline{\mathbf{D}}^{H} \overline{\mathbf{D}}+\mathbf{A}^{-1} \otimes \mathbf{I}_{K}\right)^{-1} .
\end{aligned}
$$

3) Updating error phase $\boldsymbol{\Psi}$ : The solution to the error phase $\boldsymbol{\Psi}$ can be formulated as the following maximization problem,

$$
\begin{aligned}
\hat{\boldsymbol{\Psi}}=\arg \max _{\boldsymbol{\Psi}} & \log p\left(\mathbf{s} \mid \overline{\mathbf{w}}, \mathbf{\Psi}, \beta_{0}\right) p(\mathbf{\Psi}) \\
\equiv \arg \min _{\boldsymbol{\Psi}} & \| \mathbf{s}-\mathbf{E}_{0} \mathbf{D}_{0} \mathbf{w}_{0}-\mathbf{E}_{1} \mathbf{D}_{1} \mathbf{w}_{1} \\
& -\cdots-\mathbf{E}_{K-1} \mathbf{D}_{K-1} \mathbf{w}_{K-1} \|_{2}^{2} .
\end{aligned}
$$

By taking the derivative of (34) with respect to $\Psi$, we obtain,

$$
\boldsymbol{\psi}^{(k)}=\arctan \frac{\operatorname{Im}\left(\overline{\mathbf{D}_{k} \boldsymbol{\mu}_{k}} \odot \mathbf{s}_{\backslash k}\right)}{\operatorname{Re}\left(\overline{\mathbf{D}_{k} \boldsymbol{\mu}_{k}} \odot \mathbf{s}_{\backslash k}\right)},
$$




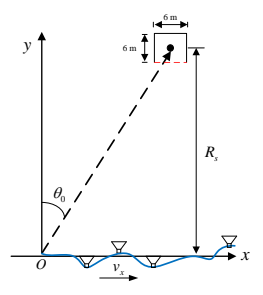

(a) (b)

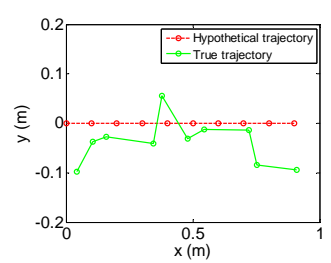

Fig. 2: Simulated geometry in TWRI and motion trajectory.

where $\boldsymbol{\mu}_{k}$. denotes the posterior mean of the reflectivity coefficients $\mathbf{w}_{k}$, and $\mathbf{s}_{\backslash k}$ is the measurement data excluding the contribution from the $k$ th propagation path.

In summary, it is observed that $\boldsymbol{\mu}$ in (19) and $\boldsymbol{\Sigma}$ in (20) are functions of $\boldsymbol{\alpha}$ in (30), $\beta_{0}$ in (31) and $\boldsymbol{\psi}$ in (35), and vice versa. It suggests an iterative approach, which iterates between (19), (20) and (30), (31) and (35), until a convergence criterion is satisfied.

\section{Simulation Results}

A typical TWRI scenario is simulated, as shown in Fig. 2(a). A transceiver mounted on a ground vehicle, moving at $v_{x}=15 \mathrm{~m} / \mathrm{s}$ along the $x$-axis, transmits and receives the stepped-frequency signal, consisting of 200 equally spaced frequency steps from $1 \mathrm{GHz}$ to $2 \mathrm{GHz}$, and the pulse repetition frequency (PRF) is $150 \mathrm{~Hz}$. The data collection time is one second, and thus the length of synthetic aperture is $1 \mathrm{~m}$ and the number of synthetic positions is 10 . The origin of the coordinate system is chosen to the initial location of the transceiver. The initial squint angle is $\theta_{0}=45^{\circ}$ and the size of illuminated room is $6 \mathrm{~m} \times 6 \mathrm{~m}$. The distance $R_{s}$ from the center of room to the $x$-axis is $50 \mathrm{~m}$. The distance between two adjacent discretized image pixels along both crossrange and downrange is 0.1 $\mathrm{m}$. The received signal comprises the direct returns and the multipath returns via secondary reflections at the three interior walls, yielding a total of $K=4$ paths. The scattering coefficients for the direct paths are drawn from $\mathcal{C N}(0,1)$, and the signal amplitudes of the multipath returns are assumed to decay with the distance. Complex white Gaussian noise is added to the measurements with a signalto-noise ratio (SNR) of $10 \mathrm{~dB}$. Only $25 \%$ frequencies are randomly selected at each transceiver location for CS-based imaging.

The first simulation is to verify the effectiveness of the proposed forward model in Eq. (9). In the above TWRI geometry, random location errors with standard deviation of $v_{x} /(2 \mathrm{PRF})$ are added at each synthetic aperture position, as depcited in Fig. 2(b), which clearly shows the deviations from the hypothesized trajectory. Fig. 3(a) shows the error bar plot with means and standard deviations of phase errors in diverse multipath propagations. The average phase errors in Fig. 3(a), which are calculated based on all the pixels in the region of interest, generally differ across multipaths reflected from interior walls and transceiver locations, when the uncertain transceiver locations are used. However, the standard deviations of phase errors are much less than $\pi / 4$ across multipath propagations, no matter the transceiver location, as shown in Fig. 3(b). Therefore, it is reasonable to consider the corrupted phase error in each multipath propagations in the forward model.

The second simulation is to verify the effectiveness of the proposed ABCS algorithm. The sparse scene, shown in Fig. 4(a), is considered. Figs. 4(b) and 4(c), respectively, show the reconstructed results from BOMP [25] with the true sparsity, and block sparse Bayesian learning approach (BSBL) [22], which only take the group sparsity into

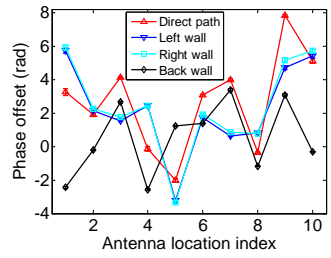

(a)

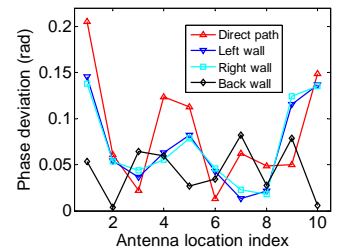

(b)
Fig. 3: Average phase errors and standard deviation. (a) Errorbar plot for phase errors. (b) Standard deviations of phase error in multipath propagations

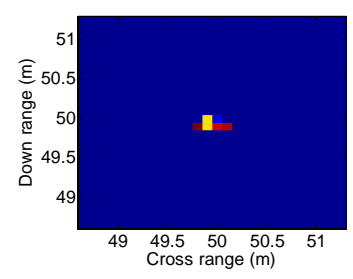

(a)

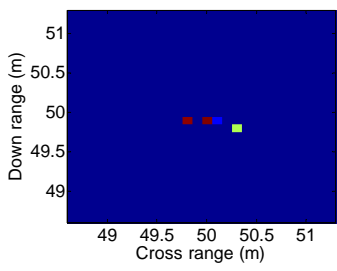

(c)

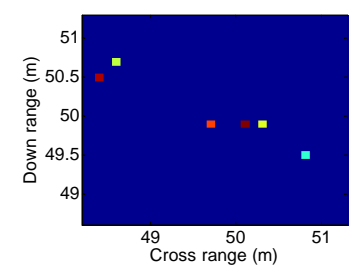

(b)

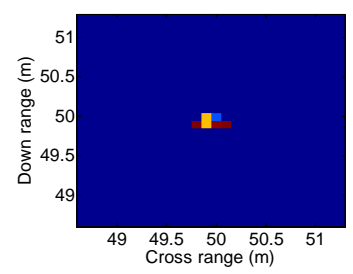

(d)
Fig. 4: Reconstructed results based on these CS approaches. (a) Original scene. (b) Result from BOMP with true sparsity. (c) Result from BSBL. (d) Result from the proposed ABCS method.

account. Due to the corrupted phase errors caused by the antenna location errors, both algorithms fail to reconstruct the sparse targets. However, by exploiting the joint sparse reconstruction and phase error correction, the proposed ABCS algorithm correctly reconstructs the sparse scene, as shown in Fig. 4(d).

\section{CONCLUSiON}

In TWRI, compressive sensing based multipath exploitation techniques enable high-resolution imaging given accurate knowledge of the physical geometry. However, the quality of the reconstructed image degrades under imperfect prior knowledge. We have prposed a novel autofocus Bayesian compressive sensing approach with multipath exploitation to perform through-the-wall scene reconstruction in the presence of uncertain transceiver locations. We first develop a forward model under multipath propagation, and then propose autofocus Bayesian compressive sensing algorithm to jointly reconstruct the sparse targets of interest and implement phase error correction in a nonparametric Bayesian framework. Simulation results are provided to validate the proposed forward model and reconstruction algorithm, and show superiority over conventional CS imaging techniques.

\section{ACKNOWLEDGMENT}

This work was supported by US Army Research Office and the Army Research Laboratory under contract W911NF-11-1-0536. 


\section{REFERENCES}

[1] M. G. Amin, Through-the-Wall Radar Imaging. CRC Press, 2010.

[2] Y. Zhang, M. G. Amin, and F. Ahmad, "A novel approach for multiple moving target localization using dual-frequency radars and time-frequency distributions," in Proc. Annual Asilomar Conf. Signals, Systems, and Computers, (Pacific Grove, CA), pp. 1817-1821, Nov. 2007.

[3] F. Ahmad and M. G. Amin, "Multi-location wideband synthetic aperture imaging for urban sensing applications," J. Franklin Inst., vol. 345, no. 6, pp. 618-639, 2008.

[4] T. Dogarur and C. Le, "Sar images of rooms and buildings based on fdtd computer models," IEEE Trans. Geosci. Remote Sens., vol. 47, no. 5, pp. 1388-1401, 2008.

[5] R. J. Burkholder, "Electromagnetic models for exploting multipath propagation in through-wall radar imaging," in Proc. Int. Conf. Electrmag. Adv. Appl., pp. 572-575, 2008.

[6] S. Kidera, T. Sakamoto, and T. Sato, "Extended imaging algorithm based on aperture synthesis with doublescattered waves for UWB radars," IEEE Trans. Geosci. Remote Sens., vol. 49, no. 12, pp. 5128-5139, 2011.

[7] P. Setlur, M. Amin, and F. Ahmad, "Multipath model and exploitation in through-the-wall and urban radar sensing," IEEE Trans. Geosci. Remote Sens., vol. 49, no. 10, pp. 4021-4034, 2011.

[8] M. Leigsnering, F. Ahmad, M. G. Amin, and A. M. Zoubir, "Compressive sensing based specular multipath exploitation for through-the-wall radar imaging," in Proc. IEEE ICASSP, (Vancouver, Canada), May 2013.

[9] Q. Wu, Y. D. Zhang, M. G. Amin, and F. Ahmad, "Throughthe-wall radar imaging based on modified Bayesian compressive sensing," in Proc. IEEE China Summit and Int. Conf. Signal and Inform. Proc., Xi'an, China, pp. 232-236, July 2014.

[10] D. E. Wahl, P. H. Eichel, D. C. Ghiglia, and C. V. Jakowatz, "Phase gradient autofocus-a robust tool for high resolution SAR phase correction," IEEE Trans. Aerospace Electron. Syst., vol. 30, no. 3, pp. 827-835, 1994.

[11] X. Li, G. Liu, and J. Ni, "Autofocusing of ISAR images based on entropy minimization," IEEE Trans. Aerospace Electron. Syst., vol. 35, no. 4, pp. 1240-1252, 1999.
[12] R. L. Morrison, M. N. Do, and D. C. Munson, "SAR image autofocus by sharpness optimization: A theoretical study," IEEE Trans. Image Proc., vol. 16, no. 9, pp. 2309-2321, 2007.

[13] N. O. Onhon and M. Cetin, "A sparsity-driven approach for joint SAR imaging and phase error correction," IEEE Trans. Image Proc., vol. 21, no. 94, pp. 2075-2088, 2012.

[14] L. Zhao, L. Wang, G. Bi, and L. Yang, "An autofocus technique for high-resolution inverse synthetic aperture radar imagery," IEEE Trans. Geosci. Remote Sens., vol. 52, no. 10, pp. 63926403, 2014.

[15] G. Wang, M. G. Amin, and Y. Zhang, "A new approach for target locations in the presence of wall ambiguity," IEEE Trans. Aerospace Electron. Syst., vol. 42, no. 1, pp. 301-315, 2006.

[16] F. Ahmad, Y. Zhang, and M. G. Amin, "Three-dimensional wideband beamforming for imaging through a single wall," IEEE Geosci. Remote Sens. Lett., vol. 5, no. 2, pp. 176-179, 2008.

[17] A. Gurbuz, J. McClellan, and W. Scott, "Compressive sensing for subsurface imaging using ground panetrating radar," Signal Processing, vol. 89, no. 10, pp. 1959-1972, 2009.

[18] M. E. Tipping, "Sparse Bayesian shrinkage and selection learning and the relevance vector machine," Journal of Machine Learning Research, vol. 1, no. 9, pp. 211-244, 2001.

[19] C. M. Bishop, Pattern Recognition and Machine Learning. New York, NY: Springer, 2006.

[20] D. J. C. MacKay, "Bayesian interpolation," Neural Comput., vol. 4, no. 3, pp. 415-447, 1992.

[21] S. Ji, Y. Xue, and L. Carin, "Bayesian compressive sensing," IEEE Trans. Signal Proc., vol. 56, no. 6, pp. 2346-2356, 2008.

[22] Z. Zhang and B. D. Rao, "Extension of SBL algorithm for the recovery of block sparse signals with inter-block correlation," IEEE Trans. Signal Proc., vol. 92, no. 7, pp. 1580-1590, 2012.

[23] P. Stoica and P. Babu, "Spice and likes: Two hyperparameterfree methods for sparse-parameter estimation," Signal Processing, vol. 92, no. 7, pp. 1580-1590, 2012.

[24] Q. Wu, Y. D. Zhang, M. G. Amin, and B. Himed, "Complex multitask Bayesian compressive sensing," in Proc. IEEE ICASSP, (Florence, Italy), May 2014.

[25] Y. C. Eldar, P. Kuppinger, and H. Bölcskei, "Block-sparse signals: Uncertainty relations and efficient recovery," IEEE Trans. Signal Proc., vol. 58, no. 6, pp. 3042-3054, 2010. 